\title{
La vida doble de las mujeres en la Ciudad de Buenos Aires
}

\section{The double life of women in the City of Buenos Aires}

\author{
Victoria Mazzeo \\ victoria.mazzeo@gmail.com \\ Instituto Gino Germani, Facultad de Ciencias Sociales, \\ Universidad de Buenos Aires, Argentina \\ Fabiana Bocchicchio \\ fabianabocchicchio@gmail.com \\ Instituto Gino Germani, Facultad de Ciencias Sociales, \\ Universidad de Buenos Aires, Argentina
}

Recepción: 09 Junio 2020

Aprobación: 02 Noviembre 2020

Publicación: 01 Marzo 2021

Citasugerida: Mazzeo, V. y Bocchicchio, F. (2021). La vida doble de las mujeres en la Ciudad de Buenos Aires. Descentrada, 5(1), e134. https://doi.org/10.24215/25457284e134
Resumen: Los abordajes para conceptualizar la categoría de género hacen referencia a las construcciones sociales y culturales de los procesos de diferenciación, dominación y subordinación entre hombres y mujeres, poniendo el énfasis en la noción de multiplicidad de identidades. Lo femenino y lo masculino se conforman a partir de una relación mutua, cultural e histórica. No se refiere al sexo de los individuos, sino a las conductas consideradas femeninas o masculinas. En el área de los estudios de familia, la perspectiva de género cuestionó la idea de que el mundo doméstico es algo exclusivo de las mujeres. El artículo cuantifica el tiempo del trabajo no remunerado intra-hogar ejercido por las mujeres y lo compara con el destinado al trabajo remunerado y al resto de actividades que desarrollan en un día. La información empírica proviene de los resultados de la Encuesta sobre Uso del Tiempo en la Ciudad de Buenos Aires 2016. Las evidencias son contundentes y confirman la desigualdad en el tiempo dedicado por los varones y mujeres en el trabajo no remunerado, y la intensidad del uso del tiempo de ellas.

Palabras clave: Género, Trabajo no remunerado, Trabajo remunerado.

Abstract: The approaches to conceptualize the gender category refer to the social and cultural constructions of the processes of differentiation, domination and subordination between men and women, emphasizing the notion of multiplicity of identities. The feminine and the masculine are formed from a mutual, cultural and historical relationship. It does not refer to the sex of the individuals, but to the behaviors considered feminine or masculine. In the area of family studies, the gender perspective questioned the idea that the domestic world is something exclusive to women. The article quantifies the time of unpaid intra-household work carried out by women and compares it with that destined to paid work and other activities that take place in one day. The empirical information comes from the results of the Survey on the Use of Time in the City of Buenos Aires 2016. The evidence is overwhelming and confirms the inequality in the time spent by men and women in unpaid work and the intensity of their use of time.

Keywords: Gender, Unpaid work, Paid work. 


\section{INTRODUCCIÓN}

Las relaciones sociales se entienden desde la perspectiva de género como relaciones de poder en todos los ámbitos: económico, social, político y cultural. La igualdad de género supone que las aspiraciones y necesidades de mujeres y varones se consideren, valoren y promuevan de igual manera. En este sentido, el enfoque de género permite identificar los diferentes papeles y tareas que llevan a cabo ambos, así como las asimetrías y las relaciones de poder e inequidades.

El concepto de género se empleó cada vez más para dar cuenta del significado decisivo de los condicionamientos sociales y culturales. "El género hacía visible la construcción histórica de los sexos, toda vez que cada cultura indicaba las funciones: las actividades y las expectativas de comportamiento relacionadas con cada uno de ellos" (Barrancos, 2008, p.3).

Para comprender el alcance teórico y metodológico de dicho concepto, es necesario plantear como marco teórico la división sexual del trabajo y la organización social que lo regula. La división sexual del trabajo separa el trabajo productivo del reproductivo de manera paradigmática y determina el lugar de mujeres y varones en la economía. En el ámbito de la familia, significa para las mujeres sumar a su trabajo de cuidado un segundo trabajo en el ámbito productivo, lo que equivale a asumir un "doble rol", una "doble jornada" o una "vida doble”. Federici (2018), en su estudio sobre el patriarcado del salario, afirma que la familia para las mujeres es esencialmente la institucionalización del trabajo no remunerado, de la dependencia salarial de los hombres y, en consecuencia, de la desigual división de poder. La organización social de los trabajos de cuidados y el lugar que ocupan en la sociedad actual son producto de un largo proceso histórico que comenzó a gestarse durante la transición al capitalismo liberal (Carrasco, Borderías y Torns, 2011).

En la Agenda 2030 para el Desarrollo Sostenible de Naciones Unidas (Organización de Naciones Unidas, 2015) se observa una especial preocupación por reconocer y valorar los trabajos de cuidados no remunerados y el trabajo doméstico mediante la prestación de servicios públicos, la promoción de infraestructura y la formulación de políticas de protección social, así como también mediante la promoción de la responsabilidad compartida en el hogar y la familia (Meta 5.4 de los Objetivos del Desarrollo Sostenible). Tal compromiso se había plasmado explícitamente en varios instrumentos internacionales, entre los que se destacan la Plataforma de Acción de la Cuarta Conferencia Mundial sobre la Mujer de Beijing (1995), la Convención para la Eliminación de todas las Formas de Discriminación contra la Mujer CEDAW (1979) y la Convención Regional del Sistema Interamericano para la Prevención, Sanción y Erradicación de la Violencia contra la Mujer (1994); convenciones aprobadas en la Argentina por las leyes 23.179 (1985) y 24.632 (1996), respectivamente.

La cuantificación estadística del trabajo no remunerado requiere una conceptualización del sistema económico capaz de incluirla de un modo adecuado a su dimensión y calidad. El contenido de dicho trabajo es el mantenimiento de los espacios y bienes domésticos, así como el cuidado a los miembros del hogar (niños y personas mayores), que incluye el apoyo escolar y/o aprendizaje, el acompañamiento y traslado, así como los cuidados temporales de salud y apoyo psicológico a los miembros de la familia.

Las encuestas de uso del tiempo son esenciales para cuantificar el trabajo de reproducción social sin remuneración que las mujeres realizan a través del trabajo doméstico no pago y el cuidado de las personas del hogar no remunerado. Estas encuestas comenzaron a implementarse en los países centrales de Europa en los años 70, y en América Latina y el Caribe a partir de la década de los 80 .

En este contexto, el artículo se propone cuantificar el trabajo no remunerado intra-hogar mayoritariamente ejercido por las mujeres en la Ciudad de Buenos Aires. La información empírica se refiere a los resultados de la Encuesta sobre Uso del Tiempo en la Ciudad de Buenos Aires (UT-CABA 2016). $\mathrm{Al}$ mismo tiempo, se pretende conjugar estos resultados con los guarismos correspondientes al resto de las actividades y al mercado de trabajo remunerado. 
En primer lugar, se presenta el estado de la cuestión, para luego presentar los antecedentes de las encuestas sobre uso del tiempo realizadas en el país. A continuación, se especifica la metodología empleada y se compara el uso del tiempo entre varones y mujeres en las actividades desarrolladas. Posteriormente, se muestra la relación entre el uso del tiempo para el trabajo no remunerado y remunerado y las características socioeconómicas de las mujeres.

\section{Estado de LA CUESTión}

En los últimos años, se produjo un importante avance en las ciencias sociales, al incorporarse los denominados estudios de la mujer como un nuevo paradigma. El género, como categoría social, es una de las contribuciones teóricas más significativas del feminismo contemporáneo.

A partir de los años 70 , se impulsó el uso de la categoría género con el objetivo de diferenciar las construcciones sociales y culturales de la biología. Ello se debe a que los roles sociales asignados y ejercidos por las mujeres y los varones, son el resultado de construcciones sociales y culturales asumidas históricamente. Además, son la consecuencia de una desigual jerarquización de las prácticas sociales, las funciones y la ubicación que se tenga en la sociedad.

Así, la categoría de género es una definición de carácter histórico y social acerca de los roles, identidades y valores que son atribuidos a varones y mujeres e internalizados mediante los procesos de socialización. La lógica del género es una lógica de poder. Se plantea como marco teórico de fondo la división sexual del trabajo y la organización social que la regula, es decir, el sistema de género que afecta a la relación entre varones y mujeres. De esta manera, las mujeres han sido impulsadas a interesarse por temas específicos relacionados con el hogar, mientras que el papel de los varones está en la vida pública (Batthyány, 2004).

Se ha afirmado que la búsqueda de soluciones a los problemas suscitados por la reproducción social, incluyendo el trabajo doméstico y de cuidados, fue uno de los núcleos conflictuales en el tránsito de la sociedad de antiguo régimen a la sociedad moderna. Es la economía feminista la que se presenta como pensamiento transformador, al proponer un nuevo paradigma que sitúa el trabajo de cuidados como aspecto determinante de la reproducción social y de las condiciones de vida de la población (Carrasco, Borderías y Torns, 2011).

Una de las primeras rupturas feministas consistió en definir las actividades no remuneradas como una dimensión del trabajo necesaria para la reproducción de la sociedad. El aspecto más interesante en el debate sobre el trabajo doméstico y, especialmente relacionado con el trabajo de cuidados, fue el desarrollo del concepto de reproducción social, surgido con especial fuerza desde el feminismo italiano. A principios de la década de 1980, Antonella Picchio fue pionera en plantear el análisis del mercado laboral desde la perspectiva de la reproducción social (Carrasco, Borderías y Torns, 2011). Es así que el trabajo doméstico integrado en el trabajo de reproducción incluye la producción de bienes materiales para el mantenimiento físico de las personas, pero también el cuidado directo de los niños y niñas y de las personas adultas, además de los afectos y de las relaciones sociales. Es decir, lo que se conoce como trabajo de cuidados.

Por su parte, Federici (2018) en su estudio sobre el trabajo no remunerado afirma que uno de los aportes más importantes a nivel teórico del movimiento feminista europeo de los años 70 fue la campaña Salario para el trabajo doméstico y la teoría del patriarcado del salario. En este sentido, la incorporación de este trabajo como parte fundamental del circuito macroeconómico ha sido una de las principales contribuciones de la economía feminista. Se incluye la economía del cuidado como aspecto relevante de la reproducción social y se devela que el trabajo doméstico y de cuidados sería absolutamente necesario para la supervivencia del trabajo de mercado.

Entonces, la economía feminista puso su énfasis en visibilizar los costos que la provisión de este trabajo reproductivo traía aparejados para las mujeres. Ya que el trabajo reproductivo era invisible para las mediciones estándares de la economía, en respuesta a esta invisibilidad surge el proyecto de contabilizar el trabajo de las 
mujeres mediante su incorporación a las cuentas nacionales y es también el origen de los esfuerzos para medir el trabajo reproductivo a través de encuestas de uso del tiempo (Esquivel, 2011b).

En sociología, los primeros estudios que contemplaron de algún modo la existencia de los cuidados se sitúan en la década de los años 80 del siglo XX, de la mano de algunas sociólogas italianas y anglosajonas. Ponían de manifiesto el trabajo invisible que las mujeres adultas realizaban para cuidar la vida en las sociedades del bienestar. Así, se pasó a valorar el trabajo realizado desde los hogares por sus propias características y por su importancia en el cuidado y bienestar de las personas.

También se ha considerado la importancia de conocer cuál era la naturaleza de los vínculos entre los varones y las mujeres dentro de la familia, en especial en relación a lo laboral. Barrère-Maurisson (1999), en su estudio sobre la división familiar del trabajo, afirma que se debe concebir a la familia como una institución social, donde se expresa la lógica de dichos vínculos que es la división sexual del trabajo, es decir una división basada en parte sobre la diferenciación sexual.

Desde la perspectiva de la sociedad patriarcal, la lógica de los vínculos sociales les atribuye un lugar secundario a las mujeres, lo que plantea un fenómeno de inferioridad en el empleo y una posición subordinada en la familia. En general, las mujeres asumen la carga del trabajo doméstico cuando la posición económica del hogar no permite pagarlos (Mazzeo, 2018). Históricamente, la división sexual del trabajo ha modelado una inserción laboral diferente de varones y mujeres: la idea del varón proveedor y la mujer cuidadora determinó brechas de género en el mercado laboral.

Sin embargo, cada grupo sexual se ve afectado simultáneamente por ambas esferas: la laboral y la familiar. Los hombres ya no se relacionan sólo con la producción y las mujeres con la familia. De esta manera, "la familia es el lugar de reparto de trabajo (profesional y doméstico) entre hombre y mujer" (Barrère-Maurisson, 1999, p. 158).

La medición del trabajo no remunerado ha generado un creciente interés en las últimas décadas. De hecho, cobró relevancia a partir de la Plataforma de Acción de Beijing, surgida de la Cuarta Conferencia Mundial de la Mujer realizada en 1995, donde se instó a los países a diseñar e implementar medios estadísticos que pudieran visibilizar el trabajo de las mujeres, incluso en el sector no remunerado y en el hogar.

En los años 70, en los países centrales de Europa, se comenzó a discutir y a establecer metodologías comunes para mostrar las relaciones sociales de género y, especialmente, cuantificar el trabajo de cuidados. A partir de los años 80, en América Latina y el Caribe, se desarrollaron encuestas de uso del tiempo a nivel nacional o en las grandes ciudades. Los estudios de uso del tiempo marcaron un punto de inflexión en el intento de hacer visible el trabajo realizado por las mujeres en el hogar y contribuyeron de forma importante a poner de relieve la estructura social del tiempo: las desigualdades en el uso del tiempo y las implicaciones del uso del tiempo en la participación laboral de mujeres y hombres.

\section{ANTECEDENTES}

En el año 2005, la Dirección General de Estadística y Censos (DGEyC), a través de la Encuesta Anual de Hogares, incorporó por primera vez dentro de sus contenidos el módulo correspondiente a la Encuesta de Uso del Tiempo de la Ciudad de Buenos Aires. Su objetivo fue brindar información sobre el tiempo que las mujeres y varones dedicaban al trabajo para el mercado, al trabajo doméstico y de cuidados no remunerados y a otras actividades relacionadas con el estudio, el tiempo libre, el descanso, entre otros.

Esta encuesta fue la primera en aplicarse en Argentina utilizando como instrumento de recolección de la información un "diario de actividades del día de ayer", administrado a través de una guía de preguntas diseñada al efecto. El cuestionario se aplicó a una persona al azar de la población comprendida entre 15 y 74 años de edad que habitaba en hogares residenciales, excluyendo villas de emergencia o inquilinatos, hoteles, pensiones y casas tomadas (DGEyC, 2007). 
La evidencia empírica de la encuesta mostró que las mujeres participaban más y dedicaban más tiempo a actividades productivas no remuneradas. Por ello, las diferencias por género en el volumen promedio del trabajo doméstico y de cuidados de personas en el hogar se explicaron tanto por la relativa menor participación de los varones como por el menor tiempo dedicado por ellos.

En el año 2010, en la ciudad de Rosario, se concretó una Encuesta del Tiempo y Voluntariado, en la que también se utilizó un "diario de actividades del día de ayer" para conocer las actividades desarrolladas por varones y mujeres y captar actividades simultáneas. Los resultados fueron comparados con los de la Ciudad de Buenos Aires, en un artículo donde se observó que en ambas ciudades los varones duplicaban el tiempo dedicado por las mujeres al trabajo de mercado, en tanto que ellas triplicaban el trabajo no remunerado. Por otro lado, el estudio dio cuenta de que las mujeres trabajaban en total más tiempo que los varones (Ganem, Giustiniani, Peinado, Geli y Andreozzi, 2018).

Con posterioridad, en el relevamiento del tercer cuatrimestre del año 2013, el Instituto Nacional de Estadística y Censos (INDEC) incorporó un módulo de Trabajo No Remunerado y Uso del Tiempo a la Encuesta Anual de Hogares Urbanos (EAHU) y produjo la primera información con cobertura nacional urbana sobre el tiempo que varones y mujeres dedicaban a estas actividades. El objetivo fue captar información respecto de la participación y el tiempo destinado por las personas de 18 años y más a las tareas domésticas, al cuidado de miembros del hogar y al trabajo voluntario, para residentes en hogares particulares de localidades de dos mil o más habitantes de todo el territorio nacional. El módulo se implementó con dos bloques, uno destinado a indagar sobre el trabajo doméstico y de cuidado no remunerado, y otro sobre trabajo voluntario. El bloque de trabajo no remunerado constó de tres preguntas, todas con período de referencia del día de ayer al relevamiento (INDEC, 2014).

Respecto a su metodología, se han efectuado algunas críticas entre las que destacan que se trató de un breve módulo montado sobre una encuesta regular a hogares; que las preguntas fueron pocas y acotadas, incluyendo un listado de tareas limitado; que la captación fue por recordación y no con el esquema de diario de actividades; que la condición de dedicar por lo menos una hora a las actividades para considerarlas como realizadas pudo producir sesgos en los tiempos según sexo y sobreestimación en los tiempos dedicados a las actividades no remuneradas; que la captación de la simultaneidad fue acotada y no explícita; que no especificaron las actividades desagregadas sino a grupos de actividades, y que al no distinguir entre días de semana y fines de semana, o entre días típicos y no típicos, pudieron distorsionarse los promedios (Rodríguez Enríquez, 2014).

Por último, en junio de 2020 el INDEC, presentó un documento de trabajo, donde informó que se encuentra trabajando desde 2019 en el diseño de la Encuesta Nacional del Uso del Tiempo, con el objetivo de producir información sobre las actividades que realizan las personas y el tiempo que le destinan (INDEC, 2020). En esta ocasión, será una encuesta específica sobre uso del tiempo y trabajo no remunerado a nivel nacional y se utilizará, para su captación, la opción del diario de actividades.

Evidentemente, la implementación de las encuestas del uso del tiempo ha sido fundamental para dar cuenta de la problemática de las desigualdades de género y la extrema intensidad en el uso del tiempo de las mujeres. Por otro lado, se considera que debe continuar siéndolo para responder con información válida a los Objetivos de Desarrollo Sostenible en el marco de la Agenda 2030 de las Naciones Unidas.

\section{Metodología}

La división sexual del trabajo se refiere a la especialización de mujeres y varones en distintos tipos de trabajos, en particular aquellos relacionados con la esfera del hogar y lo privado (el trabajo reproductivo) en el caso de las mujeres, y con el mercado y la esfera pública (el trabajo productivo), en el caso de los varones (Esquivel, 2011a). El modelo de "varón proveedor y mujer cuidadora" determinó brechas de género sostenidas en la participación en el mercado laboral. No obstante, en los últimos años se destaca el aumento sostenido de la tasa de participación femenina. 
En este sentido, el artículo analiza la brecha de género en el uso del tiempo y, en particular, en el trabajo reproductivo no remunerado de las mujeres en el hogar. El mismo incluye todas aquellas actividades que involucran la atención de los miembros del hogar, la crianza de los niños, las tareas de cocina y limpieza, el mantenimiento general del hogar y el cuidado de enfermos o personas con discapacidad. En síntesis, se refiere al conjunto de actividades realizadas en y para la esfera doméstica, con la finalidad de asegurar la reproducción cotidiana de sus miembros.

La información empírica se refiere a los resultados de la Encuesta sobre Uso del Tiempo en la Ciudad de Buenos Aires (UT-CABA 2016). En cuanto a las características de dicha encuesta, se utilizaron dos cuestionarios: uno relacionado al hogar y otro individual para uno de sus miembros. En el primero, se indagó acerca de la composición del hogar y las características socio-demográficas de sus miembros; las características habitacionales básicas; los ingresos totales del hogar; las estrategias de manutención del hogar; las ayudas recibidas por el hogar para tareas dentro del mismo y la institucionalización de alguno de sus miembros. El cuestionario individual fue respondido por un miembro del hogar de 14 años o más, seleccionado mediante una tabla aleatoria y no se admitieron reemplazos en el sorteo. Se aplicó un diario de actividades y se lo diferenció según su condición de día laboral o no laboral, y de día típico o no típico. Se pidió al entrevistado que relatara todo lo que hizo en el día anterior a la encuesta, pudiendo informar hasta 3 actividades realizadas de manera simultánea (DGEyC, 2017). Por lo tanto, el universo de este artículo está compuesto por las mujeres de 14 años y más residentes en hogares particulares de la ciudad.

Del total de viviendas seleccionadas en la muestra que salieron a campo (4.425), la tasa de efectividad fue del 60,6\%. La clasificación y codificación de actividades se realizó tomando como base la Clasificación de Actividades de Uso del Tiempo para América Latina y el Caribe (CAUTAL) en su versión mayo 2016. Los grandes grupos de actividades en que se publicaron los resultados son ocho: trabajo para el mercado; trabajo doméstico no pago; trabajo de cuidado no remunerado a miembros del hogar; servicios a la comunidad y ayudas no pagas; educación; convivencia social y actividades recreativas; uso de medios de comunicación y cuidado personal.

Se destaca que las encuestas de uso del tiempo sirven para conocer el tiempo dedicado por las personas en promedio a diferentes actividades y no específicamente para conocer cómo se distribuye el tiempo dedicado a las distintas actividades al interior del hogar. Es decir, permiten conocer el tiempo promedio que varones y mujeres destinan a distintos tipos de actividades (Mazzeo, 2018). A pesar de esta restricción se consideró importante para conocer las diferencias de género en el uso del tiempo, en especial el empleado en el trabajo no remunerado por las mujeres.

En el artículo se analiza el uso del tiempo promedio diario (lunes a domingo) según grandes grupos de actividades, con y sin simultaneidad, y se profundizan las características sociodemográficas de las mujeres en las actividades de trabajo para el mercado, de trabajo doméstico no pago y de trabajo no remunerado a miembros del hogar. Para ello se utilizan dos indicadores: tasa de participación (total de personas que realizaron una actividad en la población total, expresada en porcentaje) y tiempo promedio por participante (tiempo total que se dedica a una actividad en el total de personas que la realizaron, expresado en horas y minutos). En la Tabla 1 se presenta la composición porcentual de la población que respondió la encuesta por grupo de edad, según sexo. En su mayoría (80\%), son personas en edades potencialmente activas (14 a 64 años). Se destaca, en comparación, que las mujeres adultas mayores (65 años y más) representan 3 puntos porcentuales más que los varones. 
TABLA 1

Composición porcentual por grupo de edad y sexo de la población que respondió la encuesta. Ciudad de Buenos Aires. Año 2016

\begin{tabular}{|c|c|c|c|}
\hline Grupo etario & Total & Mujer & Varón \\
\hline Total & 100,0 & 100,0 & 100,0 \\
\hline $14-24$ & 16,6 & 15,6 & 17,9 \\
\hline $25-39$ & 27,4 & 25,4 & 29,9 \\
\hline $40-49$ & 15,7 & 17,5 & 13,6 \\
\hline $50-64$ & 19,6 & 19,5 & 19,6 \\
\hline 65 y más & 20,7 & 22,0 & 19,0 \\
\hline
\end{tabular}

Fuente: Mazzeo (2018)

La economía funciona hoy, no sólo por el aporte de la fuerza de trabajo masculina, sino también por el número creciente de mujeres que se incorporan y permanecen en el mercado de trabajo. Esto significa, en el ámbito de la familia, que las mujeres suman a su labor reproductiva, un segundo trabajo en el ámbito productivo, lo que equivale a tener una "vida doble".

Entonces, cabe preguntarse si la redistribución de roles según género que se produjo en el mundo de la producción, está siendo acompañado por una redistribución equivalente en el ámbito de la familia, en el mundo de la reproducción.

\section{LA VIDA DOBLE DE LAS MUJERES}

Según los resultados publicados por la Dirección General de Estadística y Censos del Gobierno de la Ciudad (2017), las jornadas diarias de trabajo total de las mujeres son más prolongadas que las de los varones. Ellas contribuyen más en el tiempo total (remunerado más no remunerado): $57,2 \%$ contra 42,8\% de los varones. Destinan un tiempo diario promedio de 8 horas 37 minutos frente a las 7 horas 51 minutos de los varones (Mazzeo, 2018). Como se verá, esto es así fundamentalmente por el tiempo dedicado al trabajo no remunerado intra-hogar.

Con el objeto de evidenciar la existencia de una brecha de género según el tipo de actividad que realizan diariamente, en la Tabla 2, se presenta la tasa de participación y el tiempo promedio diario (lunes a domingo, con simultaneidad) según grandes grupos de actividades. Las brechas más importantes se verifican tanto en el trabajo para el mercado como en los trabajos no remunerados (doméstico y de cuidado a miembros del hogar), mientras que en el resto son mínimas o no existen. 
TABLA 2

Uso del tiempo. Tasa de participación y tiempo promedio según grandes grupos de actividades

(con simultaneidad) por sexo. Población 14 años y más. Ciudad de Buenos Aires. Año 2016.

\begin{tabular}{|c|c|c|c|c|}
\hline \multirow[b]{3}{*}{ Grupo de actividad } & \multicolumn{4}{|c|}{ Promedio diario (lunes a domingo) } \\
\hline & \multicolumn{2}{|c|}{ Mujer } & \multicolumn{2}{|c|}{ Varón } \\
\hline & $\begin{array}{c}\text { Tasa de } \\
\text { participación }\end{array}$ & \begin{tabular}{|c|} 
Tiempo \\
promedio por \\
participante
\end{tabular} & $\begin{array}{c}\text { Tasa de } \\
\text { participación }\end{array}$ & $\begin{array}{c}\text { Tiempo } \\
\text { promedio por } \\
\text { participante }\end{array}$ \\
\hline Total & & 26,58 & & 26,13 \\
\hline Trabajo para el mercado & 42,8 & 7,59 & 56,8 & 8,52 \\
\hline Trabajo doméstico no pago & 90,8 & 3,27 & 82,0 & 1,57 \\
\hline $\begin{array}{l}\text { Servicios a la comunidad y } \\
\text { ayudas no pagas }\end{array}$ & 10,1 & 4,14 & 6,3 & 4,15 \\
\hline Educación & 17,1 & 5,16 & 15,9 & 5,51 \\
\hline $\begin{array}{l}\text { Convivencia social y } \\
\text { actividades recreativas }\end{array}$ & 86,8 & 4,18 & 90,1 & 4,14 \\
\hline $\begin{array}{l}\text { Uso de medios de } \\
\text { comunicación }\end{array}$ & 75,3 & 2,30 & 77,3 & 2,52 \\
\hline $\begin{array}{l}\text { Trabajo de cuidado no } \\
\text { remunerado a miembros del } \\
\text { hogar }\end{array}$ & 30,8 & 5,27 & 21,3 & 3,42 \\
\hline Cuidado personal & 100,0 & 11,46 & 100,0 & 11,32 \\
\hline
\end{tabular}

Fuente: Mazzeo (2018) en base a Encuesta Uso del Tiempo 2016. Tabulados Básicos.

La tasa de participación de los varones es superior en el trabajo para el mercado. Ellos registran 14 puntos porcentuales más que ellas y las mujeres le destinan aproximadamente 1 hora menos que ellos. Por su parte, las mujeres participan más y destinan más tiempo que los varones al trabajo doméstico no pago y, más aún, en el trabajo de cuidado a miembros del hogar.

Es importante resaltar que la mayor participación de las mujeres es en el trabajo doméstico no pago. Esto resulta razonable ya que en las tareas domésticas se incluyen tanto los hogares unipersonales como los multipersonales. Por lo tanto, involucra a más hogares y es mayor la participación (90,8\%). Cabe señalar que es al trabajo de cuidado a miembros del hogar, al que le dedicaban mayor tiempo promedio. Son las actividades vinculadas con el cuidado de los niños y las personas mayores; incluye el apoyo escolar y/o de aprendizaje a los niños, el acompañamiento y traslado a miembros del hogar y los cuidados temporales de salud (Mazzeo, 2018). Mientras a las tareas domésticas le dedicaban cerca de 3 horas y media, a las tareas de cuidado le destinaban 2 horas más. Cabe mencionar que, si bien con menor tiempo promedio diario, lo mismo ocurre con los varones. Ellos le dedicaban menor tiempo al trabajo doméstico (1 hora y 57 minutos) y más a las tareas de cuidado ( 3 horas y 42 minutos).

A fin de observar si existen diferencias en los tiempos promedios empleados según actividad, sin la presencia de simultaneidad, en la Tabla 3 se presenta el tiempo promedio diario "sin simultaneidad" por participante 
por día de la semana (lunes a domingo). El mismo se obtiene dividiendo el volumen del tiempo total de la población, según sexo, por el total de las personas, según sexo, que participaron en la actividad.

TABLA 3

Uso del tiempo. Tiempo promedio diario por participante según grandes grupos de actividades (sin simultaneidad) por sexo. Población 14 años y más. Ciudad de Buenos Aires. Año 2016

\begin{tabular}{|l|r|r|}
\hline \multicolumn{1}{|c|}{ Grupo de actividad } & \multicolumn{2}{c|}{$\begin{array}{c}\text { Tiempo promedio } \\
\text { diario (lunes a } \\
\text { domingo) }\end{array}$} \\
\cline { 2 - 3 } & Mujer & \multicolumn{1}{c|}{ Varón } \\
\hline Total1 & 24,00 & $\mathbf{2 4 , 0 0}$ \\
\hline Trabajo para el mercado & 7,51 & 8,48 \\
\hline Trabajo doméstico no pago & 2,58 & 1,44 \\
\hline Servicios a la comunidad y ayudas no pagas & 3,16 & 3,26 \\
\hline Educación & 5,12 & 5,47 \\
\hline Convivencia social y actividades recreativas & 3,08 & 3,12 \\
\hline Uso de medios de comunicación & 2,29 & 2,51 \\
\hline Trabajo de cuidado no remunerado a miembros del hogar & 3,43 & 2,34 \\
\hline Cuidado personal & 10,57 & 10,47 \\
\hline 1El tiempo promedio está en horas y minutos por lo que el total no suma 24 horas. \\
\hline
\end{tabular}

Fuente: elaboración propia en base a Encuesta Uso del Tiempo 2016. Tabulados Básicos.

Se destaca que la brecha de género se mantiene y que se reduce el tiempo promedio empleado en una hora aproximadamente. Para las mujeres, en las actividades de trabajo doméstico no pago, servicios a la comunidad y ayudas no pagas, convivencia social y actividades recreativas y trabajo de cuidado no remunerado a miembros del hogar. En los varones, en las mismas actividades con excepción de trabajo doméstico no pago. Evidentemente, son los grupos de actividades que permiten mayor simultaneidad. Por otro lado, se incrementa el cuidado personal y mantienen el promedio diario el trabajo para el mercado, la educación y el uso de medios de comunicación.

La DGEyC (2007) había ya mostrado con datos de 2005 que en la población de la ciudad el tiempo promedio que se dedicaba al trabajo para el mercado y para el trabajo doméstico y de cuidado no remunerado se distribuía de manera desigual según género. El 93\% de las mujeres participaban en el trabajo doméstico no pago, mientras que lo hacía el $66 \%$ de los varones. El tiempo promedio que las mujeres le dedicaban a esta actividad ( 3 horas 18 minutos) duplicaba el tiempo dedicado por los varones que participaban. Por su parte, el cuidado de niños y/o adultos del propio hogar mostraba tasas de participación más bajas que el trabajo doméstico (ya que sólo puede realizarse en hogares donde haya niños o adultos mayores/enfermos que cuidar): realizaba esta actividad casi un tercio de las mujeres y un quinto de los varones. Esto demuestra que, en más de diez años, se incrementó la participación de los varones en el trabajo doméstico, aunque no el tiempo que le dedican, y que la participación en el trabajo de cuidado se mantuvo similar.

Con las consideraciones a la metodología empleada, INDEC (2014) mostró que, en 2013, también fue mayor la dedicación a las tareas domésticas que al cuidado de personas en el hogar. En el primer caso, el 86\% de las mujeres le dedicaron en promedio 3,5 horas y en el segundo caso (donde es necesaria la presencia de 
personas para cuidar) los valores fueron más bajos: 19,6\% las mujeres y 12,3\% los varones, que les dedicaron 5,5 y 4,7 horas promedio, respectivamente. En decir, los resultados evidenciaron, en las tres encuestas, que las mujeres destinaron un tiempo sustantivamente mayor que los varones en el trabajo no remunerado.

Ahora bien, cabe preguntarse: ¿cuáles son las características sociodemográficas de las mujeres que trabajan en el mercado laboral y de las que lo hacen en las actividades intra-hogar no remuneradas?, ¿en qué se diferencian? A partir de aquí se analizan para ambos tipos de actividad algunos indicadores sociodemográficos que pretenden dar una respuesta a estos interrogantes.

\section{El uSO DEL TIEMPo DE LAS MUJERES EN EL TRABAJo NO REMUNERADO}

La organización social del cuidado es uno de los nudos básicos de la reproducción de la desigualdad y "se profundiza con el arraigo y la vigencia de hábitos culturales más tradicionales, asociados a estereotipos de género en torno al cuidado" (Rodríguez Enríquez, 2019, p. 141). Los hogares resuelven sus responsabilidades a través de su propio tiempo de trabajo no remunerado. En este sentido, como se mostró en las Tablas 2 y 3, son las mujeres las que más participan y le dedican más tiempo al trabajo doméstico no pago y de cuidado no remunerado de los miembros del hogar. Con respecto al trabajo doméstico no pago, le destinan 3 horas 27 minutos (Tabla 4).

Al observar las características de las mujeres participantes, se destaca en primer lugar que el tiempo que le dedican aumenta con la edad: sobrepasa las 3 horas a partir de los 40 años y las 4 horas luego de los 50 años. La brecha promedio entre los 25 y los 65 años y más está cercana a la hora y media, a favor de las últimas. Esto indicaría que, probablemente, las adultas mayores no trabajan en el mercado laboral o que lo hacen menos horas y, de esa manera, pueden ocuparse más tiempo de las tareas domésticas. 
TABLA 4

Tasa específica de participación y tiempo promedio diario (lunes a domingo) en actividades de trabajo doméstico no pago (con simultaneidad) según características de las mujeres. Población 14 años y más. Ciudad de Buenos Aires. Año 2016.

\begin{tabular}{|c|c|c|}
\hline $\begin{array}{l}\text { Características de la } \\
\text { participante }\end{array}$ & $\begin{array}{c}\text { Tasa específica de } \\
\text { participación }\end{array}$ & $\begin{array}{c}\text { Tiempo promedio por } \\
\text { participante }\end{array}$ \\
\hline Trabajo doméstico no pago & 90,8 & 3,27 \\
\hline \multicolumn{3}{|l|}{ Grupo de edad } \\
\hline $14-24$ & 79,3 & 1,40 \\
\hline $25-39$ & 87,3 & 2,59 \\
\hline $40-49$ & 93,8 & 3,24 \\
\hline $50-64$ & 97,0 & 4,09 \\
\hline $65 y+$ & 94,9 & 4,25 \\
\hline \multicolumn{3}{|l|}{ Nivel educativo alcanzado } \\
\hline Hasta secundario incompleto & 87,2 & 4,13 \\
\hline $\begin{array}{l}\text { Secundario completo/superior } \\
\text { incompleto }\end{array}$ & 89,9 & 3,26 \\
\hline Superior completo & 93,6 & 3,05 \\
\hline \multicolumn{3}{|l|}{ Parentesco con el jefe } \\
\hline Jefa & 94,9 & 3,30 \\
\hline Cónyuge o pareja & 93,9 & 4,02 \\
\hline Hija & 76,2 & 1,50 \\
\hline Otro & 82,7 & 3,17 \\
\hline \multicolumn{3}{|l|}{ Tipo de hogar } \\
\hline Unipersonal & 95,8 & 3,12 \\
\hline Nuclear con núcleo completo & 90,8 & 3,23 \\
\hline $\begin{array}{l}\text { Nuclear con núcleo } \\
\text { incompleto }\end{array}$ & 89,0 & 3,05 \\
\hline Resto de hogares & 87,3 & 4,08 \\
\hline \multicolumn{3}{|l|}{ Condición de actividad } \\
\hline Ocupada & 90,1 & 3,03 \\
\hline Desocupada & 98,6 & 3,17 \\
\hline Inactiva & 90,5 & 4,13 \\
\hline \multicolumn{3}{|l|}{ Quintiles de IPCF } \\
\hline $1^{\circ}$ quintil & 90,0 & 4,01 \\
\hline $5^{\circ}$ quintil & 91,1 & 2,44 \\
\hline
\end{tabular}

Fuente: elaboración propia en base a Encuesta Uso del Tiempo 2016. Tabulados Básicos.

$\mathrm{Al}$ tener en cuenta las diferencias según sus características sociodemográficas, puede afirmarse que le dedican más tiempo cuando tienen menor nivel educativo, son cónyuges o parejas, o son inactivas. También si residen en hogares con núcleos completos o en el resto de hogares (extendidos y compuestos), o en los de menores ingresos per cápita familiar. Esto probablemente se deba a distintos factores que se interrelacionan: estas mujeres no trabajan en el mercado laboral o lo hacen en ocupaciones menos calificadas en el trabajo informal, donde pueden manejar sus tiempos. Por otro lado, los hogares de menos ingresos tienen mayor cantidad de integrantes, lo que implica mayor carga de tarea doméstica (preparar la comida, limpiar la casa, lavar, planchar o arreglar la ropa, hacer las compras, etc.). Además, las oportunidades en el mercado laboral 
son más desfavorables y no les permiten derivar parte de estas actividades a espacios extra domésticos. Las mujeres de hogares con menos ingresos le dedican cerca del doble del tiempo que las que viven en los hogares con ingresos mayores.

Por su parte, las mujeres menores de 40 años, así como aquellas con nivel educativo superior completo, las ocupadas, las hijas del jefe/a del hogar, las que residen en hogares unipersonales o de mayores ingresos per cápita familiar, son las que dedican menos horas de su tiempo diario. Posiblemente, sean las que tienen menos responsabilidades en el hogar, viven solas o pueden pagar parte de estas actividades.

Donde las diferencias sociodemográficas son igualmente importantes y también la desigualdad económica, es en el trabajo de cuidado no remunerado a los miembros del hogar. Entre estas actividades se encuentra el cuidado a personas del hogar; cuidados temporales de salud; apoyo escolar; acompañamiento y traslado; apoyo, cuidado y acompañamiento y traslado a miembros con discapacidad o dependencia permanente. A ellas les dedican mayor tiempo y sus características son distintas (Tabla 5).

Se destacan algunas evidencias: las mujeres menores de 40 años o las de menor nivel educativo les dedican más tiempo. En cuanto al parentesco con el jefe del hogar, son las hijas y otras (entre los que pueden encontrarse las abuelas, tías u otras mujeres convivientes) las que conceden mayor dedicación. Respecto a la condición de actividad económica, son las desocupadas ( 4 horas diarias más) o inactivas las que destinan mayor tiempo promedio diario a las actividades de cuidado parental.

Las mujeres clasificadas como inactivas por las estadísticas laborales, en realidad se encuentran altamente activas. La mayor parte de su tiempo está dedicada al cuidado de los hijos y de otras personas integrantes del hogar. Se ha demostrado que su participación en actividades no remuneradas es tanto mayor cuanto menor son los ingresos del hogar (Rodríguez Enríquez, 2012).

$\mathrm{Al}$ tener en cuenta el tipo de hogar, se destaca el mayor tiempo promedio de los hogares no nucleares, es decir aquellos extendidos y compuestos. Poseen distinta composición y más miembros que, además del núcleo conyugal, incluyen otros familiares y/o no familiares. Puede pensarse que son hogares en los que las abuelas, primas o hermanas cuidan a los niños o a algún mayor que se encuentre enfermo o con discapacidad. 
TABLA 5

Tasa de específica de participación y tiempo promedio diario (lunes a domingo) en trabajo de cuidado no remunerado a miembros del hogar (con simultaneidad) según características de las mujeres. Población 14 años y más. Ciudad de Buenos Aires. Año 2016

\begin{tabular}{|c|c|c|}
\hline $\begin{array}{c}\text { Características de la } \\
\text { participante }\end{array}$ & $\begin{array}{c}\text { Tasa específica de } \\
\text { participación }\end{array}$ & $\begin{array}{c}\text { Tiempo promedio por } \\
\text { participante }\end{array}$ \\
\hline $\begin{array}{l}\text { Cuidado a miembros del hogar } \\
\text { no pago }\end{array}$ & 30,8 & 5,27 \\
\hline \multicolumn{3}{|l|}{ Grupo de edad } \\
\hline $14-24$ & 17,7 & 5,43 \\
\hline $25-39$ & 48,1 & 6,57 \\
\hline $40-49$ & 61,7 & 4,30 \\
\hline $50-64$ & 15,9 & 3,41 \\
\hline $65 y+$ & 9,1 & 3,55 \\
\hline \multicolumn{3}{|l|}{ Nivel educativo alcanzado } \\
\hline Hasta secundario incompleto & 25,6 & 4,50 \\
\hline $\begin{array}{l}\text { Secundario completo/superior } \\
\text { incompleto }\end{array}$ & 28,9 & 6,30 \\
\hline Superior completo & 35,8 & 4,48 \\
\hline \multicolumn{3}{|l|}{ Parentesco con el jefe } \\
\hline Jefa & 22,1 & 5,09 \\
\hline Cónyuge o pareja & 47,4 & 5,18 \\
\hline Hija & 23,6 & 6,37 \\
\hline Otro & 12,7 & 7,05 \\
\hline \multicolumn{3}{|l|}{ Tipo de hogar } \\
\hline Unipersonal & - & - \\
\hline Nuclear con núcleo completo & 39,5 & 5,26 \\
\hline Nuclear con núcleo incompleto & 32,2 & 5,20 \\
\hline Resto de hogares & 35,4 & 5,34 \\
\hline \multicolumn{3}{|l|}{ Condición de actividad } \\
\hline Ocupada & 37,6 & 5,07 \\
\hline Desocupada & 28,3 & 9,05 \\
\hline Inactiva & 19,3 & 5,42 \\
\hline \multicolumn{3}{|l|}{ Quintiles de IPCF } \\
\hline $1^{\circ}$ quintil & 46,6 & 6,06 \\
\hline $5^{\circ}$ quintil & 16,0 & 3,34 \\
\hline
\end{tabular}

Fuente: elaboración propia en base a Encuesta Uso del Tiempo 2016. Tabulados Básicos.

$\mathrm{Al}$ considerar los quintiles de ingreso per cápita familiar, se pone de relieve la desigualdad económica: en los hogares pertenecientes al $1^{\circ}$ quintil (el de menores ingresos per cápita familiar) las tasas de participación son mayores y las mujeres les dedican más tiempo que en los hogares de mayores ingresos ( $5^{\circ}$ quintil). Las que viven en hogares de menores ingresos per cápita familiar aportan algo más de 6 horas mientras las otras destinan 3 horas y media. Probablemente, ello se deba a que los hogares de mayores ingresos sean de menor tamaño, con menor demanda de cuidados o con mayores oportunidades para derivar el cuidado a instancias domésticas remuneradas.

En este sentido, se consideró importante mostrar la diferencia en la tasa de participación y en el tiempo promedio diario dedicado por las mujeres a estas actividades no remuneradas en hogares con miembros 
potencialmente demandantes de cuidado, según tenencia de asistencia para el cuidado de miembros del hogar (Tabla 6). Esta asistencia no incide en las tasas de participación en el trabajo doméstico ya que la reciben sólo para el cuidado de miembros del hogar y, como se observa, en él ambas destinan tiempos similares. Ahora bien, con respecto a los valores de las tasas de participación para el cuidado no remunerado, hay que tener en consideración una serie de factores combinados: el tipo de ayuda que se recibe (por ejemplo, si es en el propio hogar o debe llevarse al miembro a alguna institución, lo que insume tiempo para su traslado), quién la brinda (institución, un persona contratada o un familiar), el tiempo que se le dedica, así como la composición del hogar (presencia de mayores cargas de personas dependientes). El tiempo promedio dedicado muestra que a las mujeres que no tienen asistencia, les insume casi 2 horas más.

TABLA 6

Tasa de participación y tiempo promedio diario (lunes a domingo) en trabajo doméstico y de cuidado no remunerado a miembros del hogar (con simultaneidad) según tenencia de asistencia para el cuidado del hogar. Población femenina de 14 años y más residente en hogares con miembros potencialmente demandantes de cuidados. Ciudad de Buenos Aires. Año 2016.

\begin{tabular}{|c|c|c|c|c|}
\hline \multirow[b]{2}{*}{ Tenencia de asistencia } & \multicolumn{2}{|c|}{ Trabajo doméstico no pago } & \multicolumn{2}{|c|}{ Cuidado no remunerado } \\
\hline & $\begin{array}{c}\text { Tasa de } \\
\text { participación }\end{array}$ & $\begin{array}{c}\text { Tiempo } \\
\text { promedio por } \\
\text { participante }\end{array}$ & $\begin{array}{c}\text { Tasa de } \\
\text { participación }\end{array}$ & $\begin{array}{c}\text { Tiempo } \\
\text { promedio por } \\
\text { participante }\end{array}$ \\
\hline Total & 92,1 & 3,55 & 47,1 & 5,40 \\
\hline $\begin{array}{l}\text { Con asistencia para el } \\
\text { cuidado }\end{array}$ & 91,2 & 3,42 & 79,5 & 5,02 \\
\hline $\begin{array}{l}\text { Sin asistencia para el } \\
\text { cuidado }\end{array}$ & 92,6 & 4,03 & 26,3 & 6,54 \\
\hline
\end{tabular}

Fuente: elaboración propia en base a Encuesta Uso del Tiempo 2016. Tabulados Básicos.

Estas evidencias dan cuenta de la injusta organización social del cuidado, que descansa sobre el trabajo no remunerado de las mujeres e implica un uso muy intensivo de su tiempo, aún mayor para el cuidado parental.

\section{El USO DEL TIEMPo DE LAS MUJERES EN EL TRABAJO REMUNERAdo}

Un aspecto relevante del mercado de trabajo en el siglo XXI es la progresiva incorporación de las mujeres. Los datos de la Ciudad de Buenos Aires muestran que, entre 2004 y 2016, se redujo la brecha entre las tasas de actividad y de empleo según sexo. "Se destacan los mayores incrementos en las tasas de actividad (4 puntos porcentuales) y de empleo ( 3 puntos porcentuales) de las mujeres, mientras que los varones en igual período sólo las aumentan en 1 punto porcentual" (Mazzeo y Bocchicchio, 2019, p.81). En los años 60 tan solo 3 de cada 10 mujeres declaraban ser parte de la fuerza laboral; medio siglo después, 5 de ellas se concentran en esta condición (Mazzeo y Bocchicchio, 2017). Los niveles de las tasas de actividad y de empleo en 2016 de las mujeres llegaron a 58\% y 52,3\%, respectivamente.

El aumento de la participación de las mujeres en el mercado de trabajo se hizo sin gastos públicos y sin servicios sociales suficientes para apoyar las tareas de cuidado intra-hogar, sobre todo de las familias de bajos recursos. Esto llevó a que muchas mujeres pasaran a tener una vida doble (trabajo productivo y reproductivo) o que no lograran insertarse en el trabajo productivo debido a la alta carga de cuidado en el 
hogar, especialmente en hogares pobres que no podían mercantilizar los cuidados. Esto no ha hecho más que reforzar la desigualdad entre géneros.

Cuando se profundiza el análisis del uso del tiempo en el trabajo para el mercado (Tabla 7), se constata que las mujeres registran mayores tasas de participación laboral y tiempo promedio diario entre los $25 \mathrm{y}$ 64 años o en el nivel educativo superior completo, donde superan las 8 horas diarias en promedio. Esto encuentra explicación en el corrimiento de la edad a la unión, mayores credenciales educativas e inserción en el mercado de trabajo, que trajeron como consecuencia un retraso en la formación de la familia y en la procreación (Mazzeo, 2018). Asimismo, se destaca que las mayores participaciones las ostentan quienes son jefas o cónyuges o parejas. Esto es lógico ya que en el primer caso se incluyen todos los hogares (incluyendo los unipersonales), mientras que en el segundo caso sólo los conyugales.

Al tener en cuenta el tipo de hogar, las mayores participaciones se registran en los hogares nucleares con núcleo completo (pareja conyugal, con o sin hijos solteros), en los unipersonales y en los nucleares incompletos o monoparentales (sin uno de los miembros de la pareja conyugal con hijos solteros). Debe tenerse en cuenta el alto peso relativo femenino en este último ya que las mujeres, cuando finaliza una unión, quedan a cargo de sus hijos. En 2016, el 85\% de los hogares monoparentales tenían jefatura femenina, mientras que para el total de hogares era sólo el 46\% (Mazzeo, 2018). Es evidente que las diferencias en el tiempo promedio no son muy notorias, la amplitud entre los valores extremos no supera los 35 minutos. No obstante, se destaca la mayor participación de las mujeres en núcleos incompletos, en los cuales seguramente son la única o principal proveedora.

Al considerar la desigualdad económica, a través de los quintiles de ingresos per cápita familiar extremos, se destaca que en los hogares con menos ingresos, en comparación, las tasas de participación son menores (en 21 puntos porcentuales). Asimismo, el tiempo promedio dedicado al mercado de trabajo es menor (cerca de 50 minutos) que en los hogares con más ingresos. Probablemente, esté relacionado con el trabajo informal que es más común en los hogares con menores ingresos y la mayor carga intra-hogar de trabajo no remunerado. 
TABLA 7

Tasa específica de participación y tiempo promedio diario (lunes a domingo) en actividades de trabajo para el mercado (con simultaneidad) según características de las mujeres. Población 14 años y más. Ciudad de Buenos Aires. Año 2016.

\begin{tabular}{|c|c|c|}
\hline Características de la participante & $\begin{array}{c}\text { Tasa especifica de } \\
\text { participación }\end{array}$ & $\begin{array}{l}\text { Tiempo promedio por } \\
\text { participante }\end{array}$ \\
\hline Trabajo para el mercado & 42,8 & 7,59 \\
\hline \multicolumn{3}{|l|}{ Grupo de edad } \\
\hline $14-24$ & 19,7 & 7,07 \\
\hline $25-39$ & 59,1 & 8,21 \\
\hline $40-49$ & 62,4 & 8,05 \\
\hline $50-64$ & 52,0 & 8,19 \\
\hline $65 y+$ & 16,8 & 5,58 \\
\hline \multicolumn{3}{|l|}{ Nivel educativo alcanzado } \\
\hline Hasta secundario incompleto & 23,2 & 7,54 \\
\hline $\begin{array}{l}\text { Secundario completo/superior } \\
\text { incompleto }\end{array}$ & 36,7 & 7,48 \\
\hline Superior completo & 60,1 & 8,07 \\
\hline \multicolumn{3}{|l|}{ Parentesco con el jefe } \\
\hline Jefa & 49,8 & 8,10 \\
\hline Cónyuge o pareja & 44,9 & 7,57 \\
\hline Hija & 28,1 & 7,37 \\
\hline Otro & 20,5 & 6,24 \\
\hline \multicolumn{3}{|l|}{ Tipo de hogar } \\
\hline Unipersonal & 39,8 & 7,59 \\
\hline Nuclear con núcleo completo & 44,4 & 8,08 \\
\hline Nuclear con núcleo incompleto & 49,8 & 7,56 \\
\hline Resto de hogares & 36,9 & 7,33 \\
\hline \multicolumn{3}{|l|}{ Quintiles de IPCF } \\
\hline $1^{\circ}$ quintil & 37,7 & 7,32 \\
\hline $5^{\circ}$ quintil & 59,1 & 8,19 \\
\hline
\end{tabular}

Fuente: elaboración propia en base a Encuesta Uso del Tiempo 2016. Tabulados Básicos.

Se consideró importante observar, para los hogares con presencia de miembros potencialmente demandantes, la tasa de participación y el tiempo promedio diario dedicado al mercado de trabajo, según la tenencia de asistencia en el hogar (Tabla 8). Se destaca que las mujeres que no tienen asistencia para el cuidado de personas en el hogar registran una participación laboral que es menor a la mitad de las que sí la tienen, y en cuanto al tiempo dedicado es una hora menor. 
TABLA 8

Tasa de participación y tiempo promedio diario (lunes a domingo) en actividades de trabajo para el mercado (con simultaneidad) según tenencia de asistencia para el cuidado del hogar. Población femenina de 14 años y más residente en hogares con miembros potencialmente demandantes de cuidados. Ciudad de Buenos Aires. Año 2016.

\begin{tabular}{|l|c|c|}
\hline \multicolumn{1}{|c|}{ Tenencia de asistencia } & Tasa de participación & $\begin{array}{c}\text { Tiempo promedio por } \\
\text { participante }\end{array}$ \\
\hline Total & $\mathbf{3 5 , 6}$ & $\mathbf{7 , 2 1}$ \\
\hline $\begin{array}{l}\text { Con asistencia para el } \\
\text { cuidado }\end{array}$ & 53,1 & 7,44 \\
\hline Sin asistencia para el cuidado & 24,3 & 6,48 \\
\hline
\end{tabular}

Fuente: elaboración propia en base a Encuesta Uso del Tiempo 2016. Tabulados Básicos.

Evidentemente, lo que sucede en el mercado laboral está explicado por lo que pasa en materia de distribución de responsabilidades de cuidado dentro del hogar. Por otro lado, existe una brecha sustantiva entre las mujeres en asociación directa a su posición en la estructura social. "El mayor peso del trabajo no remunerado sobre las mujeres con menores recursos económicos es un determinante clave de su menor y peor inserción laboral" (Rodríguez Enríquez, 2019, p.146).

A pesar del aumento de las mujeres en la participación en el mercado de trabajo, las desigualdades domésticas siguen siendo muy fuertes. Cuando se considera la totalidad de los tiempos (remunerado, doméstico, parental, personal, social, etc.) las mujeres aparecen como perdedoras. Siguiendo la lógica de clasificación de los tiempos, que compara, por jornadas de 24 horas, los tiempos promedio consagrados sin simultaneidad (Tabla 9), se observa que a las mujeres les quedan 9 horas 28 minutos para lo extra-laboral y a los varones cerca de 11 horas. La mayor diferencia se encuentra en el tiempo dedicado al trabajo doméstico y parental, en el que se destaca que ellas le dedican 2 horas y media más que ellos.

TABLA 9

Tiempo total promedio por participante (sin simultaneidad) por sexo según grupo de actividad (día promedio de lunes a domingo). Población de 14 años y más. Ciudad de Buenos Aires. Año 2016.

\begin{tabular}{|l|c|c|c|c|}
\hline \multicolumn{1}{|c|}{ Grupo de actividad } & \multicolumn{2}{c|}{ Mujer } & \multicolumn{2}{c|}{ Varón } \\
\hline Total $^{1}$ & $\mathbf{2 4 , 0 0}$ & $\mathbf{1 0 0 , 0}$ & $\mathbf{2 4 , 0 0}$ & $\mathbf{1 0 0 , 0}$ \\
\hline Trabajo para el mercado & 7,51 & 32,7 & 8,48 & 36,7 \\
\hline $\begin{array}{l}\text { Trabajo doméstico y cuidado } \\
\text { miembros del hogar }\end{array}$ & 6,41 & 27,8 & 4,18 & 17,9 \\
\hline Extra-laboral & 9,28 & 39,5 & 10,54 & 45,4 \\
\hline \multicolumn{4}{|l|}{ El tiempo promedio está en horas minutos por lo que el total no suma 24 horas. } \\
\hline
\end{tabular}

Fuente: elaboración propia en base a Encuesta Uso del Tiempo 2016. Tabulados Básicos.

La situación se agrava aún más si se tienen en cuenta solo los hogares con miembros potencialmente demandantes de cuidado y se considera la existencia de ayuda paga (Tabla 10). 
TABLA 10

Hogares con miembros potencialmente demandantes de cuidado. Tiempo total promedio por participante (sin simultaneidad) por sexo según grupo de actividad (día promedio de lunes a domingo). Población de 14 años y más. Ciudad de Buenos Aires. Año 2016.

\begin{tabular}{|l|c|c|c|c|c|c|c|c|}
\hline \multirow{2}{*}{ Grupo de actividad } & \multicolumn{4}{|c|}{ Mujer } & \multicolumn{4}{c|}{ Varón } \\
\cline { 2 - 10 } & \multicolumn{2}{|c|}{ Con ayuda } & \multicolumn{2}{|c|}{ Sin ayuda } & \multicolumn{2}{c|}{ Con ayuda } & \multicolumn{2}{c|}{ Sin ayuda } \\
\hline Total1 & $\mathbf{2 4 , 0 0}$ & $\mathbf{1 0 0 , 0}$ & $\mathbf{2 4 , 0 0}$ & $\mathbf{1 0 0 , 0}$ & $\mathbf{2 4 , 0 0}$ & $\mathbf{1 0 0 , 0}$ & $\mathbf{2 4 , 0 0}$ & $\mathbf{1 0 0 , 0}$ \\
\hline Trabajo para el mercado & 7,44 & 32,2 & 6,48 & 28,3 & 9,17 & 38,7 & 7,52 & 32,8 \\
\hline $\begin{array}{l}\text { Trabajo doméstico y cuidado } \\
\text { miembros del hogar }\end{array}$ & 8,44 & 36,4 & 10,57 & 45,6 & 5,03 & 21,0 & 7,12 & 30,0 \\
\hline Extra-laboral & 7,32 & 31,4 & 6,15 & 26,1 & 9,40 & 40,3 & 8,56 & 37,2 \\
\hline
\end{tabular}

${ }^{1}$ El tiempo promedio está en horas y minutos por lo que el total no suma 24 horas.

Fuente: elaboración propia en base a Encuesta Uso del Tiempo 2016. Tabulados Básicos.

El trabajo no remunerado de las mujeres con ayuda paga es menos intenso que en aquellas que no la tienen (más de 2 horas menos). No obstante, es mayor en 1 hora el remunerado. Como consecuencia, es mayor el tiempo extra-laboral que poseen. Al observar el comportamiento de los varones, se destacan también diferencias importantes según la existencia de ayuda paga. No obstante, si se compara la intensidad del uso del tiempo según sexo y existencia de ayuda paga, se destaca que en el trabajo no remunerado ellas, exista o no ayuda paga, destinan más de 3 horas y media que los varones.

Como consecuencia, la disparidad afecta otros aspectos de la equidad de las mujeres: el tiempo adicional que las mujeres pasan realizando labores domésticas, sobre todo en relación con los niños, es una causa principal de las brechas de género en los sueldos y los ascensos en el trabajo. Las mujeres siguen padeciendo la segregación horizontal y vertical del mercado laboral. Por otro lado, esta situación las ha llevado a reducir las horas de descanso y de ocio personal.

\section{Conclusiones}

El exceso de trabajo no remunerado de las mujeres, su vida doble, persiste a pesar del incremento sostenido de las tasas de participación en el mercado laboral registrado en las últimas décadas. En los países de América Latina y el Caribe, la existencia de información, obtenida de forma más sistemática sobre el uso del tiempo y el trabajo no remunerado, así lo evidencia. Las responsabilidades de los cuidados están distribuidas desigualmente: "Responden a un modelo familiar, con una participación limitada del Estado, con una oferta de mercado limitada y segmentada y con una provisión comunitaria insuficiente y segregada. Además, en el seno de los hogares, y como consecuencia de la división sexual del trabajo, las mujeres asumen o se les impone el rol de cuidadoras" (CEPAL, 2019, p.144).

La importancia de las labores realizadas dentro del hogar para satisfacer las necesidades biológicas y afectivas ha estado siempre estrechamente relacionada a la esfera femenina, pues sobre las mujeres recae el cuidado de niños, enfermos y adultos mayores.

El concepto de economía del cuidado ha permitido analizar las actividades de trabajo no remunerado en su escala más micro, mediante la observación de las labores fundamentales para la reproducción de la fuerza de trabajo que se realizan en el seno de los hogares. Los hallazgos obtenidos permiten confirmar que las mujeres trabajan en total un tiempo sustantivamente mayor que los varones. Esto es debido al tiempo empleado en las tareas domésticas y de cuidado no remuneradas que desarrollan dentro de sus hogares. 
La vida doble de las mujeres es más intensa en las edades centrales, en los menores niveles educativos, cuando son cónyuges o parejas, son inactivas y más bajo es el ingreso per cápita del hogar en el que viven. Las brechas de género más importantes se verifican tanto en el trabajo para el mercado, como en los trabajos no remunerados (doméstico y de cuidado a miembros del hogar). En comparación, los varones muestran mayor dedicación en el trabajo remunerado, y las mujeres en el trabajo doméstico y de cuidado a miembros del hogar.

Es importante resaltar que es al trabajo de cuidado a miembros del hogar al que le dedican las mujeres mayor tiempo promedio, ya que son las actividades vinculadas con el cuidado de los niños y las personas mayores; incluye el apoyo escolar y/o de aprendizaje a niños, el acompañamiento y traslado a miembros del hogar y los cuidados temporales de salud.

Por su parte, en los hogares de menores ingresos per cápita, las tasas de participación son mayores y les dedican más tiempo que en los hogares de mayores ingresos. Probablemente, ello se deba a que los hogares de mayores ingresos sean de menor tamaño, con menos demanda de cuidados o con mayores oportunidades para derivar el cuidado a instancias domésticas remuneradas. La situación se agrava aún más si se tienen en cuenta sólo los hogares con miembros potencialmente demandantes de cuidado y se considera la existencia de ayuda paga.

En síntesis, la evidencia empírica es contundente y confirma la desigualdad en el tiempo dedicado por los varones y mujeres en el trabajo no remunerado y en la intensidad del uso del tiempo de ellas. Las responsabilidades domésticas aparecen como una tensión para las mujeres, que deben resolver ajustando los tiempos de trabajo en el mercado, descanso y esparcimiento. Evidentemente, esto tiene implicancias en su calidad de vida.

Se enfatiza la relevancia y utilidad de este tipo de información, para dar cuenta de una problemática incuestionable en la determinación de las desigualdades de género y en las condiciones de vida de las mujeres.

Ante los cambios demográficos, socioeconómicos y medioambientales que pueden aumentar la demanda de cuidados y sin políticas públicas adecuadas, podrían incrementarse las brechas de género e incluso podría agudizarse la crisis de los cuidados. El modelo actual de organización social de los cuidados, que se basa en las familias y se mantiene gracias al trabajo no remunerado de las mujeres, ya no es sostenible.

\section{REFERENCIAS}

Barrancos, Dora (2008). Mujeres entre la casa y la plaza. Buenos Aires: Ed. Sudamericana.

Barrère-Maurisson, M. A. (1999). La división familiar del trabajo. La vida doble. Ciudad de Buenos Aires: Ed. Lumen Humanitas.

Batthyány, K. (2004). Cuidado infantil y trabajo: ¿Un desafio exclusivamente femenino? una mirada desde el género y la ciudadanía social. Montevideo: Cinterfor. Recuperado de https://www.oitcinterfor.org/sites/default/files/fi le_publicacion/trazos_20.pdf

Carrasco C., Borderías C. y Torns T. (2011). Introducción. El trabajo de cuidados: Antecedentes históricos y debates actuales. En Carrasco C., Borderías C. y Torns T. (Eds.) El trabajo de cuidados. Historia, teoría y politicas. 13-96. Madrid: Los libros de la Catarata. Recuperado de https://www.fuhem.es/media/cdv/file/biblioteca/Economia _critica/El_trabajo_de_cuidados_C._Carrasco_C._Borderias_T._Torns.pdf

CEPAL (2019). Economía del cuidado: tendencias e impactos del cambio demográfico. En CEPAL La autonomía de las mujeres en escenarios económicos cambiantes. 132-174. Santiago: Impreso en Naciones Unidas. Recuperado de https://www.cepal.org/es/publicaciones/45032-la-autonomia-mujeres-escenarios-economicos-cambiantes

Dirección General de Estadística y Censos (2007). El tiempo de trabajo total. Mujeres y varones en la Ciudad de Buenos Aires. Encuesta Anual de Hogares 2005. Uso del tiempo. Informe de Resultados $N^{\circ} 328$. DGEyC. Recuperado de https://www.estadisticaciudad.gob.ar/eyc/?p=37531 
Dirección General de Estadística y Censos (2017). Encuesta sobre Uso del Tiempo en la Ciudad de Buenos Aires UTCABA 2016. Objetivos, marco conceptual y aspectos metodológicos. Buenos Aires: DGEyC-GCBA. Recuperado de https://www.estadisticaciudad.gob.ar/eyc/?p=71834

Dirección General de Estadística y Censos (2017). Encuesta Uso del Tiempo 2016. Tabulados Básicos. Ciudad Autónoma de Buenos Aires. Recuperado de https://www.estadisticaciudad.gob.ar/eyc/wpcontent/uploads/20 17/12/ut2016_tabulados_basicos.pdf

Esquivel, V. (2011a), Introducción: Hacer economía feminista desde América Latina. En Esquivel, V. (coord.) La economía feminista desde América Latina: Una hoja de ruta sobre los debates actuales en la región. (pp.24-41). Santo Domingo: Grupo Género y Macroeconomía América Latina. Recuperado de http://www.gemlac.org/at tachments/article/44/Economia-feminista-desde-america-latina.pdf

Esquivel, V. (2011b) La economía del cuidado en América Latina: Poniendo a los cuidados en el centro de la agenda. Ciudad de Panamá: Área Práctica de Género. Serie Atando Cabos; deshaciendo nudos. Recuperado de https:// www.americalatinagenera.org/es/documentos/Atando_Cabos.pdf

Federici, S. (2018). El patriarcado del salario. Ciudad Autónoma de Buenos Aires: Tinta Limón.

Ganem, J., P. Giustiniani, G. Peinado, M. Geli y L. Andreozzi (2018). Estudio comparativo de los usos del tiempo en las ciudades de Rosario y Buenos Aires, Argentina. En Población de Buenos Aires Año 15 N$^{\circ} 27.31-48$. Ciudad de Buenos Aires.

INDEC (2014). Encuesta sobre trabajo no remunerado y uso del tiempo. Resultados por Jurisdicción. Buenos Aires: INDEC. Informe de Prensa. Julio. Recuperado de https://www.indec.gob.ar/uploads/informesdeprensa/tnr_ 07_14.pdf

INDEC (2020). Hacia la Encuesta Nacional sobre Uso del Tiempo y Trabajo No Remunerado. Documento de trabajo INDEC N ${ }^{\circ} 30$. Buenos Aires: INDEC. Recuperado de https://www.indec.gob.ar/ftp/cuadros/sociedad/docu mento_trabajo_enut.pdf

Mazzeo, V. (2018). Las diferencias de género en el uso del tiempo y el trabajo no remunerado en la Ciudad de Buenos Aires. En XIII Jornadas Nacionales de Debate interdisciplinario en Saludy Población. IGG -Facultad de Ciencias Sociales, Universidad de Buenos Aires. Ciudad de Buenos Aires, Argentina. Recuperado de: http://jornadassal udypoblacion.sociales.uba.ar/ponencias/mesa-9-cuidados-familia-y-salud/

Mazzeo, V. y F. Bocchicchio (2017). La brecha laboral de género en la Ciudad de Buenos Aires. En: IV Seminario Internacional Desigualdad y Movilidad Social en América Latina. Ciudad de La Plata. Recuperado de http://se minariosms.fahce.unlp.edu.ar/sdyms-2017/actas/MazzeoBocchicchio.pdf/view

Mazzeo, V. y F. Bocchicchio (2019). Las mujeres y el envejecimiento de la población total y activa de la Ciudad de Buenos Aires. Revista Población y Desarrollo: argonautas y caminantes, 15, 76-85. doi: https://doi.org/10.5377 /pdac.v15i0.8119

Organización de Naciones Unidas (1996). Informe de la Cuarta Conferencia Mundial sobre la Mujer. Recuperado de: https://www.un.org/womenwatch/daw/beijing/pdf/Beijing\%20full\%20report\%20S.pdf

Organización de Naciones Unidas (2015). Transformar nuestro mundo: la Agenda 2030 para el Desarrollo Sostenible. Resolución aprobada por la Asamblea General el 25 de septiembre de 2015. Recuperado de: https://www.un.org/ $\mathrm{ga} /$ search/view_doc.asp?symbol=A/RES/70/1\&Lang=S

Rodríguez Enríquez, C. (2012). La cuestión del cuidado: ¿El eslabón perdido del análisis económico? En Revista CEPAL, 106, 23-36. Santiago de Chile: CEPAL. Recuperado de https://www.cepal.org/es/publicaciones/373 65-revista-cepal-no106

Rodríguez Enríquez, C. (2014). El trabajo de cuidado no remunerado en Argentina: un análisis desde la evidencia del Módulo de Trabajo no Remunerado. En: Serie Documentos de Trabajo "Políticas públicas y derecho al cuidado". $\mathrm{N}^{\circ}$ 2. 1-24. Equipo Latinoamericano de Justicia y Género. Recuperado de https://ri.conicet.gov.ar/handle/11 $336 / 34802$

Rodríguez Enríquez, C. (2019). Aportes de la economía feminista para abordar la desigualdad: la cuestión del cuidado. En Quiroga Díaz N. y P. Dobrée (Comps.) Luchas y alternativas para una economía feminista emancipatoria. 
140-151. CABA: CLACSO. Montevideo: AFM. Asunción: CDE. Recuperado de http://biblioteca.clacso.edu .ar/clacso/gt/20191108021928/Luchas_y_alternativas.pdf 